\title{
The Explicit Construction of Einstein Finsler Metrics with Non-Constant Flag Curvature ${ }^{\star}$
}

\author{
Enli $\mathrm{GUO}^{\dagger}$, Xiaohuan $M \mathrm{O}^{\ddagger}$ and Xianqiang $Z H A N G \S$ \\ $\dagger$ College of Applied Science, Beijing University of Technology, Beijing 100022, China \\ E-mail: guoenli@bjut.edu.cn \\ $\ddagger$ Key Laboratory of Pure and Applied Mathematics, School of Mathematical Sciences, \\ Peking University, Beijing 100871, China \\ E-mail:moxh@pku.edu.cn \\ $\S$ Tianfu College, Southwestern University of Finance and Economics, Mianyang 621000, China \\ E-mail: zxq@tf-swufe.net
}

Received December 08, 2008, in final form April 09, 2009; Published online April 14, 2009

doi:10.3842/SIGMA.2009.045

\begin{abstract}
By using the Hawking Taub-NUT metric, this note gives an explicit construction of a 3-parameter family of Einstein Finsler metrics of non-constant flag curvature in terms of navigation representation.
\end{abstract}

Key words: Finsler manifold; Einstein Randers metric; Ricci curvature

2000 Mathematics Subject Classification: 58E20

\section{Introduction}

The Ricci curvature of a Finsler metric $F$ on a manifold is a scalar function Ric : $T M \rightarrow \mathbb{R}$ with the homogeneity $\operatorname{Ric}(\lambda y)=\lambda^{2} \operatorname{Ric}(y)$. See (3) below. A Finsler metric $F$ on an $n$-dimensional manifold $M$ is called an Einstein metric if there is a scalar function $K=K(x)$ on $M$ such that

$$
\text { Ric }=(n-1) K F^{2} \text {. }
$$

Recently, C. Robles studied a special class of Einstein Finsler metrics, that is, Einstein Randers metrics and obtained the following interesting result [1, Proposition 12.9]: Let $F$ be a Randers metric on a 3-dimensional manifold. Then $F$ is Einstein if and only if it has constant flag curvature. Note that the flag curvature in Finsler geometry is a natural extension of the sectional curvature in Riemannian geometry and it furnishes the lowest order term for the Jacobi equation that governs the second variation of geodesics of the Finsler metric. Together with the classification theorem of Randers metrics of constant flag curvature due to Bao-Robles-Shen [2], three dimensional Einstein Randers metrics are completely determined.

The Randers metrics were introduced by physicist Randers in 1941 by modifying a Riemannian metric $\alpha:=\sqrt{a_{i j}(x) y^{i} y^{j}}$ by a linear term $\beta:=b_{i}(x) y^{i}[8]$. By requiring $\|\beta\|_{\alpha}<1$, we ensure that $\alpha+\beta$ is positive and strongly convex. The interested reader is referred to [1] for this result and a thorough treatment of Randers metrics.

The next problem is to describe four dimensional Einstein Randers metrics. This problem turns out to be very difficult. The very first step might be to construct as many examples as possible. In 2002 D. Bao and C. Robles constructed for the first time a family of non-constant

\footnotetext{
*This paper is a contribution to the Special Issue "Élie Cartan and Differential Geometry". The full collection is available at http://www.emis.de/journals/SIGMA/Cartan.html
} 
flag curvature Einstein Randers metrics on $\mathbb{C} P^{2}$ using Killing vector fields with respect to its Fubini-Study metric [1].

The main technique in [1] is described as follows. Given a Riemannian metric $g=g_{i j}(x) y^{i} y^{j}$ and a vector field $W=W^{i} \frac{\partial}{\partial x^{i}}$ on a manifold $M$ with $g\left(x, W_{x}\right)<1$, one can define a Finsler metric $F: T M \rightarrow[0, \infty)$ by

$$
F(x, y)=\sqrt{g}\left(x, y-F(x, y) W_{x}\right) .
$$

Solving (1) for $F$, one obtains $F=\alpha+\beta$, where $\alpha=\sqrt{a_{i j}(x) y^{i} y^{j}}$ and $\beta=b_{i}(x) y^{i}$ are given by

$$
a_{i j}=\frac{g_{i j}}{\lambda}+\frac{W_{i} W_{j}}{\lambda^{2}}, \quad b_{j}=-\frac{W_{j}}{\lambda},
$$

where $W_{i}=g_{i j} W^{j}$ and $\lambda=1-W_{i} W^{i}$.

In this paper, using (1) we are going to construct a 3-parameter family of Einstein Randers metrics with non-constant flag curvature.

Now let us describe our construction. Let $\left(N^{3}, h\right)$ be an oriented constant curvature 3manifold, set $M^{4}=\mathbb{R} \times N^{3}$, and let $\varphi: M^{4} \rightarrow N^{3}$ be the projection onto the second factor. Define a Riemannian metric $g$ on $M^{4}$ by

$$
g=u \varphi^{*} h+u^{-1}(d t+A)^{2},
$$

where $u$ is a positive smooth function and $A$ a 1 -form on $N^{3}$. Then $\left(M^{4}, g\right)$ is Einstein if and only if $u$ and $A$ are related by the monopole equation of mathematical physics

$$
d u=-* d A
$$

and $\left(N^{3}, h\right)$ is flat, in which case $g$ is Ricci-flat, that is, $g$ has zero Ricci curvature $[4,5]$. For $a \geq 0$, define a harmonic function on $\mathbb{R}^{3} \backslash\{0\}$ by

$$
u_{a}(y)=\frac{1}{4}\left(\frac{1}{|y|}+a\right) .
$$

Then the above construction gives the Hawking Taub-NUT Riemannian metric $g_{a}(a>0)$ or the standard metric $g_{0}(a=0)$. Note that the metric $g_{a}$ extends to the whole of $\mathbb{R}^{4}$; in fact it is given by the explicit formula $[4,5,7,11]$

$$
g_{a}=\left(a|x|^{2}+1\right) g_{0}-\frac{a\left(a|x|^{2}+2\right)}{a|x|^{2}+1}\left(-x^{2} d x^{1}+x^{1} d x^{2}-x^{4} d x^{3}+x^{3} d x^{4}\right)^{2} .
$$

See [5] for discussion of $g_{1}$. For any $m, n \in \mathbb{R}^{+}$, we define $W_{m, n} \in \Gamma\left(T \mathbb{R}^{4}\right)$ by

$$
W_{m, n}=-m x^{2} \frac{\partial}{\partial x^{1}}+m x^{1} \frac{\partial}{\partial x^{2}}-n x^{4} \frac{\partial}{\partial x^{3}}+n x^{3} \frac{\partial}{\partial x^{4}} .
$$

Let $\Omega:=\left\{x \in \mathbb{R}^{4} \mid f(x)<1\right\}$, where $f(x)$ is defined in (21). We obtain the following result:

Theorem 1. Let $F=\sqrt{a_{i j}(x) y^{i} y^{j}}+b_{i}(x) y^{i}$ be any function in $T \Omega \rightarrow[0, \infty)$ on $T \Omega$ which is expressed by (2) in terms of the Hawking Taub-NUT metric $g_{a}$ and vector field $W_{m, n}$. Then $F$ has the following properties:

(i) F is a Randers metric;

(ii) F is Einstein with Ricci constant zero;

(iii) F has non-constant flag curvature.

Our main approach is to show $W_{m, n}$ is the vector field induced by a one parameter isometric group with respect to the Hawking Taub-NUT metric $g_{a}$.

These examples show the existence of a large family of global Einstein Finsler metrics on $\mathbb{R}^{4}$ (taking $m=n<a$, then $\Omega=\mathbb{R}^{4}$ ). This is still far from a complete description, of course, but it gives an indication that this family is much larger than previously believed. 


\section{Preliminaries}

Let $F$ be a Finsler metric on an $n$-dimensional manifold $M$. The second variation of geodesics gives rise to a family of endomorphisms $R_{y}=R_{k}^{i} d x^{k} \otimes \frac{\partial}{\partial x^{i}}: T_{x} M \rightarrow T_{x} M$, defined by

$$
R_{k}^{i}=2 \frac{\partial G^{i}}{\partial x^{k}}-y^{j} \frac{\partial^{2} G^{i}}{\partial x^{j} \partial y^{k}}+2 G^{j} \frac{\partial^{2} G^{i}}{\partial y^{j} \partial y^{k}}-\frac{\partial G^{i}}{\partial y^{j}} \frac{\partial G^{j}}{\partial y^{k}},
$$

where $G^{i}$ are the geodesic coefficients of $F[6,10] . F$ is said to be of constant flag curvature $K=\lambda$, if $R_{k}^{i}=\lambda\left(F^{2} \delta_{k}^{i}-F F_{y_{k}} y^{i}\right)$ where $F_{y^{k}}:=\frac{\partial F}{\partial y^{k}}$ [6]. Finsler metrics of constant flag curvature are the natural extension of Riemannian metrics of constant sectional curvature.

The Ricci curvature Ric is defined to be the trace of $R_{y}$

$$
\operatorname{Ric}(y):=R_{k}^{k}(x, y) \text {. }
$$

Ric is a well-defined scalar function on $T M \backslash\{0\} . F$ is called an Einstein metric if there is a scalar function $K=K(x)$ on $M$ such that Ric $=(n-1) K F^{2}$.

Consider a Randers metric $F=\alpha+\beta$ on $M$. Let $g=g_{i j} y^{i} y^{j}$ be the Riemannian metric and $W=W^{i} \frac{\partial}{\partial x^{i}}$ be the vector field on $M$ such that $F$ is defined by [1]. Then D. Bao and C. Robles showed the following result $[1,9]$ :

Proposition 1. F is Einstein with Ricci scalar $\operatorname{Ric}(x):=(n-1) K(x)$ if and only if

(i) $W$ satisfies $W_{i \mid j}+W_{j \mid i}=-4 c g_{i j}$, where $W_{i}=g_{i j} W^{j}$, and

(ii) $g$ is an Einstein metric, i.e. $\widetilde{\text { Ric }}=(n-1)\left\{K(x)+c^{2}\right\} g$, where $c=$ const and $\widetilde{\text { Ric }}$ is the Ricci curvature tensor of $g$, in particular $K(x)=$ const if $n \geq 3$.

\section{One parameter transformation group}

We rewrite the Hawking Taub-NUT metric $g_{a}$ as

$$
g_{a}=g_{i j} d x^{i} d x^{j}
$$

where

$$
\left(g_{i j}\right)=G(x)=\left(\begin{array}{cccc}
B-A\left(x^{2}\right)^{2} & A x^{1} x^{2} & -A x^{2} x^{4} & A x^{2} x^{3} \\
A x^{1} x^{2} & B-A\left(x^{1}\right)^{2} & A x^{1} x^{4} & -A x^{1} x^{3} \\
-A x^{2} x^{4} & A x^{1} x^{4} & B-A\left(x^{4}\right)^{2} & A x^{3} x^{4} \\
A x^{2} x^{3} & -A x^{1} x^{3} & A x^{3} x^{4} & B-A\left(x^{3}\right)^{2}
\end{array}\right)
$$

and

$$
B=B(x)=a|x|^{2}+1, \quad A=A(x)=a\left(1+\frac{1}{B}\right) .
$$

For any $m, n \in \mathbb{R}^{+}$, we define $\phi: \mathbb{R}^{4} \rightarrow \mathbb{R}^{4}$ by

$$
\phi_{\theta}=\left(\phi_{\theta}^{1}, \phi_{\theta}^{2}, \phi_{\theta}^{3}, \phi_{\theta}^{4}\right), \quad\left(\begin{array}{c}
y^{1} \\
y^{2} \\
y^{3} \\
y^{4}
\end{array}\right)=\phi_{\theta}^{T}=A_{\theta}\left(\begin{array}{c}
x^{1} \\
x^{2} \\
x^{3} \\
x^{4}
\end{array}\right)
$$

where $A_{\theta}$ is given by

$$
A_{\theta}=\left(\begin{array}{cc}
A_{\theta, m} & , 0 \\
0 & A_{\theta, n}
\end{array}\right)
$$


and

$$
A_{\theta, m}=\left(\begin{array}{cc}
\cos (m \theta) & -\sin (m \theta) \\
\sin (m \theta) & \cos (m \theta)
\end{array}\right), \quad A_{\theta, n}=\left(\begin{array}{cc}
\cos (n \theta) & -\sin (n \theta) \\
\sin (n \theta) & \cos (n \theta)
\end{array}\right) .
$$

It is easy to see that

$$
|y|^{2}:=\sum_{i=1}^{4}\left(y^{i}\right)^{2}=\sum_{i=1}^{4}\left(x^{i}\right)^{2}=|x|^{2} .
$$

Furthermore, $\phi_{\theta}$ is a one-parameter transformation group.

\section{Killing fields}

In this section, we explicitly construct a two-parameter family of Killing fields of the Hawking Taub-NUT Riemannian metrics. First, we prove that $\phi_{\theta}$ in (6) is an isometry. It is equivalent to prove that

$$
A_{\theta}^{T} G(y) A_{\theta}=G(x)
$$

Let

$$
H(x):=\left(\begin{array}{cc}
H_{1}(x) & H_{2}(x) \\
H_{2}^{T}(x) & H_{3}(x)
\end{array}\right)
$$

where

$$
\begin{aligned}
& H_{1}(x):=\left(\begin{array}{c}
x^{2} \\
-x^{1}
\end{array}\right)\left(x^{2},-x^{1}\right), \quad H_{2}(x):=\left(\begin{array}{c}
x^{2} \\
-x^{1}
\end{array}\right)\left(x^{4},-x^{3}\right), \\
& H_{3}(x):=\left(\begin{array}{c}
x^{4} \\
-x^{3}
\end{array}\right)\left(x^{4},-x^{3}\right) .
\end{aligned}
$$

It follows from (4), (11) and (12) that

$$
G(x)=B(x) I-A(x) H(x) .
$$

Plugging (9) into (5) yields

$$
A(x)=A(y), \quad B(x)=B(y) .
$$

Note that $A_{\theta}$ satisfies that

$$
A_{\theta}^{T}=A_{\theta}^{-1} \text {. }
$$

By using (13), (14) and (15), we obtain that (10) is equivalent to

$$
H(x)=A_{\theta}^{T} H(y) A_{\theta} .
$$

In order to check (16), from (7), (8) and (11), it is enough to check 3 matrix equations of order $2 \times 2$ as follows:

$$
H_{1}(x)=A_{\theta, m}^{T} H_{1}(y) A_{\theta, m}, \quad H_{2}(x)=A_{\theta, m}^{T} H_{2}(y) A_{\theta, n}, \quad H_{3}(x)=A_{\theta, n}^{T} H_{3}(y) A_{\theta, n},
$$


where $A_{\theta, m}$ and $A_{\theta, n}$ are defined in (8). Hence

$$
A_{\theta, m}^{T} H_{1}(y) A_{\theta, m}=A_{\theta, m}^{T}\left(\begin{array}{c}
y^{2} \\
-y^{1}
\end{array}\right)\left(y^{2},-y^{1}\right) A_{\theta, m}=\left(\begin{array}{c}
x^{2} \\
-x^{1}
\end{array}\right)\left(x^{2},-x^{1}\right)=H_{1}(x),
$$

thus we obtain the first equation of (17), the others are completely analogous.

For any fixed $p=\left(p^{1}, p^{2}, p^{3}, p^{4}\right) \in \mathbb{R}^{4}$, we have

$$
\begin{aligned}
& \frac{d \phi_{\theta}^{1}(p)}{d \theta}=-m\left(p^{1} \sin (m \theta)+p^{2} \cos (m \theta)\right)=-m \phi_{\theta}^{2}(p), \\
& \frac{d \phi_{\theta}^{2}(p)}{d \theta}=m\left(p^{1} \cos (m \theta)-p^{2} \sin (m \theta)\right)=m \phi_{\theta}^{2}(p) .
\end{aligned}
$$

Similarly, we have

$$
\frac{d \phi_{\theta}^{3}(p)}{d \theta}=-n \phi_{\theta}^{3}(p), \quad \frac{d \phi_{\theta}^{4}(p)}{d \theta}=n \phi_{\theta}^{4}(p) .
$$

It follows that the vector field induced by $\phi_{\theta}$ is given by

$$
\begin{aligned}
W_{p} & =\left.\frac{d}{d \theta}\left[\phi_{\theta}(p)\right]\right|_{\theta=0}=\left.\left.\frac{d \phi_{\theta}^{j}(p)}{d \theta}\right|_{\theta=0} \frac{\partial}{\partial x^{j}}\right|_{p} \\
& =-\left.m p^{2} \frac{\partial}{\partial x^{1}}\right|_{p}+\left.m p^{1} \frac{\partial}{\partial x^{2}}\right|_{p}-\left.n p^{4} \frac{\partial}{\partial x^{3}}\right|_{p}+\left.n p^{3} \frac{\partial}{\partial x^{4}}\right|_{p} .
\end{aligned}
$$

Note that $\phi_{\theta}$ is an isometry. It follows that $W$ is of Killing type with respect to the Hawking Taub-NUT metric $g_{a}$.

\section{Construction of Einstein-Finsler metrics}

We rewrite our Killing field $W$ as

$$
W=W_{m, n}=\sum_{j=1}^{4} W^{j} \frac{\partial}{\partial x^{j}} .
$$

Then, from (18), we have

$$
W^{1}=-m x^{2}, \quad W^{2}=m x^{1}, \quad W^{3}=-n x^{4}, \quad W^{4}=n x^{3} .
$$

Together with (4) we get

$$
W_{j}=g_{j i} W^{i}= \begin{cases}W^{j}\left(B-\frac{\sigma A}{m}\right), & j=1,2, \\ W^{j}\left(B-\frac{\sigma A}{n}\right), & j=3,4,\end{cases}
$$

where

$$
\sigma:=m\left(x^{1}\right)^{2}+m\left(x^{2}\right)^{2}+n\left(x^{3}\right)^{2}+n\left(x^{4}\right)^{2}
$$

and $A$ and $B$ are defined in (5). It follows that

$$
\lambda=1-|W|^{2},
$$


where

$$
|W|^{2}=\sum_{j=1}^{4} W_{j} W^{j}=\left[\left(W^{1}\right)^{2}+\left(W^{2}\right)^{2}\right]\left(B-\frac{\sigma A}{m}\right)+\left[\left(W^{3}\right)^{2}+\left(W^{4}\right)^{2}\right]\left(B-\frac{\sigma A}{n}\right) .
$$

We are going to find the sufficient condition producing Einstein-Finsler metrics in terms of navigation representation, i.e. $|W|<1$.

Put

$$
p:=\max \{m, n\}, \quad q:=\min \{m, n\}, \quad \text { then } \quad p-q=|m-n| .
$$

By (19), we obtain

$$
\frac{\sigma}{m} \geq \frac{q}{p}|x|^{2}, \quad \frac{\sigma}{n} \geq \frac{q}{p}|x|^{2} .
$$

Thus we have

$$
\begin{aligned}
& {\left[\left(W^{1}\right)^{2}+\left(W^{2}\right)^{2}\right]\left(B-\frac{\sigma A}{m}\right)+\left[\left(W^{3}\right)^{2}+\left(W^{4}\right)^{2}\right]\left(B-\frac{\sigma A}{n}\right)} \\
& \quad \leq p|x|^{2}\left(1+a|x|^{2}-\frac{a q}{p}|x|^{2} \frac{2+a|x|^{2}}{1+a|x|^{2}}\right) \\
& \quad=\frac{|x|^{2}}{1+a|x|^{2}}\left(p+2 a|m-n||x|^{2}+a^{2}|m-n||x|^{4}\right):=f(x) .
\end{aligned}
$$

Proof of Theorem 1. From (20) and (21), we obtain that $f(x)<1$ implies $\left|W_{m, n}\right|<1$. It ensures that $\left(g_{a}, W_{m, n}\right)$ produces Randers metrics in terms of navigation representation. It is

easy to check that $\sum_{i=1}^{4} \tilde{F}_{x^{i} y^{1}} y^{i} \neq \tilde{F}_{x^{1}}$, where $\tilde{F}:=\sqrt{g_{i j}(x) y^{i} y^{j}}$ and $g_{i j}=g_{a}\left(\frac{\partial}{\partial x^{i}}, \frac{\partial}{\partial x^{j}}\right)$. According to [3], $g_{a}$ is not locally projectively flat. Recall that a Finsler metric $\bar{F}$ on a $k$-dimensional manifold $M$ is projectively flat if every point of $M$ has a neighborhood $U$ that can be embedded into $\mathbb{R}^{k}$ in such a way that it carries the $\bar{F}$-geodesics in $U$ to straight line segments. In the Riemannian case, by a theorem of Bonnet-Beltrami, projective flatness is equivalent to having constant sectional curvature. Hence $g_{a}$ does not have constant sectional curvature. Note that $F:=\alpha+\beta$ has constant flag curvature if and only if $g_{a}$ has constant sectional curvature and $W_{m, n}$ is a homothetic vector field [2]. It follows that $F$ does not have constant flag curvature and we obtain ( iii) of Theorem 1. Note that the Hawking Taub-NUT metric $g_{a}$ on $\mathbb{R}^{4}$ is an Einstein metric for all $a>0[7,11]$. From Section 4 we see that $W_{m, n}$ is of Killing type with respect to $g_{a}$ for all $(m, n) \in\left(\mathbb{R}^{+}, \mathbb{R}^{+}\right)$. Now $(i i)$ of Theorem 1 is an immediate consequence of Proposition 1.

\section{References}

[1] Bao D., Robles C., Ricci and flag curvatures in Finsler geometry, in A Sampler of Riemann-Finsler Geometry, Math. Sci. Res. Inst. Publ., Vol. 50, Cambridge Univ. Press, Cambridge, 2004, 197-259.

[2] Bao D., Robles C., Shen Z., Zermelo navigation on Riemannian manifolds, J. Differential Geom. 66 (2004), 377-435, math.DG/0311233.

[3] Hamel G., Über die Geometrieen in denen die Geraden die Kürzesten sind, Math. Ann. 57 (1903), 231-264.

[4] Hawking S.W., Gravitational Instantons, Phys. Lett. A 60 (1977), 81-83.

[5] LeBrun C., Complete Ricci-flat Kähler metrics on $C^{n}$ need not be flat, in Several Complex Variables and Complex Geometry, Part 2 (Santa Cruz, CA, 1989), Proc. Sympos. Pure Math., Vol. 52, Part 2, Amer. Math. Soc., Providence, RI, 1991, 297-304. 
[6] Mo X., An introduction to Finsler geometry, Peking University Series in Mathematics, Vol. 1, World Scientific Publishing Co. Pte. Ltd., Hackensack, NJ, 2006.

[7] Pantilie R., Harmonic morphisms with 1-dimensional fibres on 4-dimensional Einstein manifolds, Comm. Anal. Geom. 10 (2002), 779-814.

[8] Randers G., On an asymmetric metric in the four-space of general relativity, Phys. Rev. 59 (1941), 195-199.

[9] Robles C., Einstein metrics of Randers type, Ph.D. Thesis, British Columbia University, Canada, 2003.

[10] Shen Z., Projectively flat Randers metrics of constant curvature, Math. Ann. 325 (2003), 19-30.

[11] Wood J.C., Harmonic morphisms between Riemannian manifolds, in Modern Trends in Geometry and Topology, Cluj Univ. Press, Cluj-Napoca, 2006, 397-414. 